\title{
Laser Therapy in the Treatment of Diabetic Retinopathy and Diabetic Macular Edema
}

\author{
Lesley A. Everett ${ }^{1}$ (D) $\cdot$ Yannis M. Paulus ${ }^{1}$ (i) \\ Accepted: 1 June 2021 / Published online: 6 September 2021 \\ (C) The Author(s), under exclusive licence to Springer Science+Business Media, LLC, part of Springer Nature 2021
}

\begin{abstract}
Purpose of Review This review highlights indications and evidence on laser therapy in the management of diabetic retinopathy and diabetic macular edema. Particular focus is placed upon the benefits and limitations of conventional laser photocoagulation versus more modern laser photocoagulation techniques, as well as the role of laser photocoagulation in treatment of diabetic retinopathy and diabetic macular edema with the frequent utilization of pharmacologic, including anti-vascular endothelial growth factor (VEGF), therapy.

Recent Findings Laser photocoagulation remains the gold-standard therapy for the effective, definitive treatment of PDR, and also is highly effective in the management of DME. However, numerous recent studies have demonstrated the clinical efficacy and improved functional and anatomic outcomes of combination therapy with pharmacologic treatment.

Summary Continuing innovations in laser technology and improved understanding of laser-retinal interactions and pathophysiology demonstrate that laser therapy will continue to play a critical role in the treatment of diabetic retinopathy and diabetic macular edema for many years to come.
\end{abstract}

Keywords Diabetic retinopathy $\cdot$ Diabetic macular edema $\cdot$ Retinal laser therapy $\cdot$ Panretinal photocoagulation $\cdot$ Focal laser photocoagulation $\cdot$ Selective retinal therapy

\section{Introduction}

Laser (Light Amplification by Stimulated Emission of Radiation) therapy is utilized widely in nearly all fields of medicine including ophthalmology, particularly in the treatment of retinal vascular diseases such as proliferative diabetic retinopathy (PDR), diabetic macular edema (DME), retinal vein occlusions, central serous chorioretinopathy, choroidal neovascularization, and vascular tumors [1]. Retinal laser therapy is used for the management of these conditions in an

This article is part of the Topical collection on Microvascular Complications-Retinopathy

Yannis M. Paulus

ypaulus@med.umich.edu

Lesley A. Everett

lesleyae@umich.edu

1 Department of Ophthalmology and Visual Sciences, Kellogg Eye Center, University of Michigan, 1000 Wall Street, Ann

Arbor, MI 48105, USA ambulatory clinic setting, as well as in the operating room as part of the surgical management for complex conditions like tractional retinal detachments in diabetic retinopathy.

The purpose of this review is to specifically highlight the indications and evidence for the role of laser therapy in the management of diabetic retinopathy and diabetic macular edema. Diabetes is the leading cause of new cases of blindness in adults in the USA [2,3]. This article focuses on the use of peripheral scatter retinal laser (e.g., panretinal photocoagulation, or PRP) to treat PDR and the use of macular focal or grid laser photocoagulation to treat DME. Although a comprehensive review of the history and development of ophthalmic lasers is beyond the scope of this article, this information is summarized in several recent reviews $[4,5]$. Similarly, the use of retinal laser therapy for other indications, such as for choroidal neovascularization, choroidal tumors, and the treatment of retinal tears or holes, is reviewed elsewhere $[6,7]$.

Since the discovery and implementation of argon laser with emission in the blue and green spectrum range by Bridges in 1964 [8], retinal laser therapy has been utilized as the standard of care for PDR. More recently, it has been used by some providers in combination with intravitreal injections of anti- 
vascular endothelial growth factor (VEGF) agents for the management of PDR. However, there is significant controversy about the use of anti-VEGF agents as a mainstay of PDR treatment, given the risk of progressive disease, tractional retinal detachment, or neovascular glaucoma if treatment is interrupted or if a patient is lost to follow up as can frequently occur in patients with DM. Retinal laser therapy also has an important role in some patients for the treatment of DME, often resulting in highly effective treatment of visually significant macular edema without the requirement of frequent recurrent intravitreal anti-VEGF injections or associated endophthalmitis risk.

Laser technology has improved and evolved over the last several decades in order to maintain important therapeutic treatment effects, while minimizing collateral tissue damage, complications, and patient discomfort. Since it represents a critical and highly effective therapy for the treatment and prevention of blinding eye disease, this article provides an update on the current role and considerations for retinal laser therapy in diabetic retinopathy in the age of pharmacologic, including anti-VEGF, treatments.

\section{How Does Retinal Laser Therapy Treat Diabetic Eye Disease?}

The principle of retinal laser therapy resulting in therapeutic effects in the target retinal tissue is based upon the absorption of light by ocular pigments, predominantly in the retinal pigment epithelium (RPE) and choroid $[9,10]$ melanin and hemoglobin. Conventional photocoagulation results in permanent chorioretinal scars, although some of the newer laser modalities utilizing reduced intensity and pulse duration may not have such permanent tissue effects, based upon studies in animal models [11]. The differences between these laser treatment types will be reviewed later in this article.

The exact mechanism by which retinal laser therapy results in effective treatment and improvement of retinal vascular disease is not fully understood. With regards to PRP for PDR, one possible mechanism is that damage to the retinal cells by laser photocoagulation in areas of poor retinal perfusion decreases the overall retinal oxygen demand and the level of retinal hypoxia, with subsequent downregulation of angiogenic factors and VEGF production by the retinal tissue and subsequent increased oxygen perfusion to the remaining viable retina $[12,13]$. Photoreceptors are the most metabolically active and numerous cell type within the retina, and PRP treatment involves the purposeful destruction of a fraction of photoreceptors in the peripheral retina to reduce overall oxygen demand. The resulting decrease in VEGF production by the retina also results in decreased retinal vascular permeability and retinal edema [14].
In contrast to the diffuse peripheral retinal treatment applied in PRP, focal laser is an approach in which laser is specifically applied to a limited area of the posterior pole to reduce macular edema. Although the exact mechanism of focal laser is also unknown, it has been proposed that focal laser may work by occluding leaking microaneurysms in the retina, followed by RPE recovery and the stimulation of cytokine production that leads to reabsorption of fluid in the macula [15]. It has also been proposed that reduced retinal tissue following photocoagulation leads to changes in retinal vascular autoregulation and a resulting decrease in retinal blood flow and macular edema [16, 17], or that reduced retinal blood flow and macular edema results from improved oxygenation after laser treatment [16]. Several studies have reported that grid treatment alone (without focal treatment of leaking microaneurysms) has a beneficial effect in the treatment of diabetic macular edema, suggesting that there is some component of a beneficial indirect effect of retinal photocoagulation on macular edema [18-21].

\section{Conventional Photocoagulation}

Conventional laser photocoagulation has numerous applications in the treatment of retinal disease, including diabetic retinopathy, retinal vein occlusions, sickle cell retinopathy, and retinal tears. For the purpose of treating diabetic retinopathy, it is most often used to administer panretinal photocoagulation of the peripheral retina, and can be delivered through a slit lamp system utilizing a contact lens [22], laser indirect ophthalmoscope (LIO) [23], or endolaser intra-operatively [24]. All of these systems utilize a laser light source connected to the output device through a fiber optic cable. Typical laser settings for conventional retinal photocoagulation utilize pulse durations from 100 to 200 milliseconds (ms), laser spot diameters from 100 to 500 micrometers (um), and powers from 100 to 750 milliwatts (mW) with the application of 1000 to 2000 medium-intensity burns in the peripheral retina, spaced onehalf to one spot width apart [25]. These parameters are titrated to produce visible gray-white burns in the treatment tissue, and variation in each of the parameter settings has direct effects on the final retinal burns produced. A complete PRP treatment can be divided into two or three treatment sessions to minimize side effects and patient discomfort.

Conventional retinal photocoagulation has several significant possible side effects and disadvantages, including patient discomfort during treatment, permanent retinal scarring, prolonged time for the physician to complete the treatment (sometimes over multiple sessions), possible choroidal detachments after treatment, elevated intraocular pressure, cystoid macular edema, and decreased patient peripheral, color, and night vision [26-28]. Direct treatment of retinal blood vessels or retinal neovascularization may result in 
hemorrhage. Although rare, misdirected light can also result in burns of the cornea, iris, lens, and fovea [29]. Anterior segment burns with the laser indirect ophthalmoscope (LIO) result from poor focus. Burns of the iris may result in iritis, accommodative difficulties, or posterior synechiae [30]. Delayed complications of photocoagulation include secondary choroidal neovascularization, subretinal fibrosis, and macular pucker, particularly in an area where laser treatment may have resulted in rupture of Bruch's membrane.

The Diabetic Retinopathy Study (DRS) was the first large, prospective, multi-center, randomized clinical trial of the efficacy of retinal laser photocoagulation, specifically to evaluate the timing of PRP in eyes with advanced non-proliferative diabetic retinopathy and with PDR [31]. This trial demonstrated that PRP was highly effective and reduced the risk of severe visual loss by $60 \%$ at 2 years in patients with high-risk PDR [31, 32]. It also demonstrated that PRP applied with argon laser had a similar clinical efficacy, but a much better adverse effect profile, as compared to xenon-arc treatment, which led to the adoption of argon laser as the most common conventional laser source utilized for PRP following that study. However, argon lasers have mostly been replaced by air-cooled Nd:YAG lasers (such as Pattern Scanning Lasers using frequency-doubled neodymium-doped yttrium aluminum garnet (Nd:YAG) laser technology, described later in this article for application of panretinal, focal, and macular grid photocoagulation) that similarly are able to produce green (532-nm) light given the smaller size and footprint of the laser device. According to the DRS protocol for standard argontype laser PRP, the laser settings should be a pulse duration of $100 \mathrm{~ms}$, large spot size of 200-500 um, and power of 200$300 \mathrm{~mW}$ utilized to deliver $1500-5000$ burns over $1-4$ treatment sessions, with each laser spot applied one by one [31].

Macular edema is the main cause of decreased vision in diabetic patients, and conventional laser photocoagulation has an important role in the treatment of this condition [33]. Macular edema can be defined as focal or diffuse, and the laser approach utilized to treat it depends on the type of macular edema. Focal macular edema is characterized by discrete areas of retinal thickening associated with specific points of leakage on fluorescein angiography. Diffuse macular edema is characterized by widespread thickening and diffuse leakage of fluorescein dye that reflects extensive breakdown of the blood-retinal barrier.

With regards to the use of conventional laser photocoagulation for the treatment of diabetic macular edema, the Early Treatment Diabetic Retinopathy Study (ETDRS) was one of the earliest prospective, multi-center, randomized clinical trials to demonstrate the efficacy of focal (direct/grid) laser therapy for the treatment of clinically significant macular edema (CSME) [25, 34]. The ETDRS definition of CSME was based on the presence of any one of the following three characteristics [33]: (1) Retinal thickening within 500 um of the center of the macula, (2) Hard exudate within 500 um of the center of the macula with associated thickening, or (3) Zone or zones of thickening larger than one disc area in size, any part of which is within one disc diameter of the center of the macula. Investigators in this study used a fluorescein angiogram to help direct laser photocoagulation treatment of DME and to identify treatable lesions, defined as discrete angiographic points of retinal hyperfluorescence or clinical points of focal leakage between 500 and 3,000 um from the center of the fovea considered to produce retinal thickening or hard exudates [35]. Two methods of laser photocoagulation were utilized: focal (to treat focal areas of leakage) or grid-pattern (to treat diffuse retinal thickening secondary to diffuse leakage) $[36,37]$. Focal treatment consists of burns of 50 to 100 um of moderate intensity and 0.05 to 0.1 second duration, with endpoint of treatment as whitening or darkening of focal lesions. Grid treatment utilizes spot size of 50 to 200 um for a duration of 0.05 to 0.5 seconds, not placed within 500 um of the center of the macula or within $500 \mathrm{um}$ of the disc margin, with treatment goal of mild retinal pigment epithelium whitening. This study demonstrated that patients with mild to moderate non-proliferative diabetic retinopathy and macular edema benefit from focal/grid laser photocoagulation with an associated reduction in the incidence of vision loss by $50 \%$ after 3 years of follow-up, relative to untreated control subjects [25, 35-37].

Although the ETDRS photocoagulation protocol was found to be very effective, the placement of retinal laser burns close to the center of the macula has the risk of progressive RPE and retinal atrophy ("laser creep") that enlarge over time and can extend into the fovea, with possible resulting loss of central vision, central scotoma, decreased color vision, choroidal neovascularization, and subretinal fibrosis $[38,39]$. To prevent this adverse outcome, altered approaches to utilize laser burns that are lighter and less intense than those used in the ETDRS protocol have been developed [40].

The resulting modified Early Treatment Diabetic Retinopathy Study direct/grid photocoagulation protocol (mETDRS) entails treating only areas of thickened retina and areas of retinal nonperfusion, as well a direct photocoagulation of leaking microaneurysms [41]. It was modified from the original ETDRS protocol in two main components: (1) there was not a requirement for a treatment-induced change in microaneurysm color, and (2) laser burns in the mETDRS protocol were less intense (gray) and smaller (50 microns) compared to the original ETDRS protocol [42].

Another approach, known as the mild macular grid (MMG) protocol, utilized the application of mild, widely spaced burns throughout the macula in areas of normal and thickened retina, but excluding the foveal region and without direct laser photocoagulation of microaneurysms [41]. The modified ETDRS direct/grid protocol was directly compared to the mild macular grid laser photocoagulation strategy for the treatment of 
diabetic macular edema in a randomized control trial [41]. The mild macular grid laser protocol was considered to be a potentially milder (but more extensive) laser technique in which microaneurysms were not treated directly, and small burns were placed throughout the macula, whether or not macular edema was present. In this study, 263 subjects with previously untreated diabetic macular edema were randomly assigned to receive laser photocoagulation either by the modified ETDRS (162 eyes) or MMG (161) protocol, with clinical outcomes (visual acuity, fundus photographs, and OCT) obtained at baseline and at 3.5, 8, and 12-month follow-up. At 12 months after treatment, the MMG technique was found to be less effective at reducing OCT-measured retinal thickening as compared to the modified ETDRS protocol, although visual acuity outcome was not significantly different between the two methods [41].

In eyes with CSME and PDR requiring immediate PRP for proliferative disease, it is generally best to deliver focal treatment before or at the same time as the PRP, rather than after $\mathrm{PRP}$, in order to minimize the risk of PRP exacerbating the macular edema. An alternate approach used commonly today is also to treat with an initial intravitreal anti-VEGF injection to temporize the proliferative manifestations and reduce the CSME, followed shortly by PRP therapy.

\section{Modern Scanning Laser Photocoagulation}

As laser technology has evolved and improved over the last several decades, emphasis has been placed on developing modifications to conventional retinal laser therapy in order to minimize retinal damage and adverse side effects, while maintaining the excellent therapeutic effect of the conventional approach. To this end, most of the innovations have focused on changing the laser pulse duration, wavelength, and spot size to achieve these goals.

For example, the semi-automated pattern scanning retinal photocoagulation system (PASCAL $®$, PAttern SCAn Laser) represents a modern method of retinal photocoagulation which enables the rapid application of numerous spots (4 to 56 burns) in a defined pattern to reduce treatment time, increase patient comfort, and improve the accuracy of treatment using a scanning laser with shorter pulse durations of $10-30 \mathrm{~ms}$ [43]. A 532-nm wavelength is utilized through a standard slit-lamp system, and a number of laser scanning patterns are available (arc, grid, circle, etc.) which may be utilized according to the patient's retinal anatomy and clinical indication (Figure 1). Through a number of histologic studies, it has been shown that such shorter pulse duration burns result in less tissue damage to the inner retina [44], as compared to the longer duration $(>100 \mathrm{~ms})$ laser burns that affect the RPE, photoreceptors, inner nuclear layer, ganglion layer, and nerve fiber layer $[45,46]$. In addition, shorter

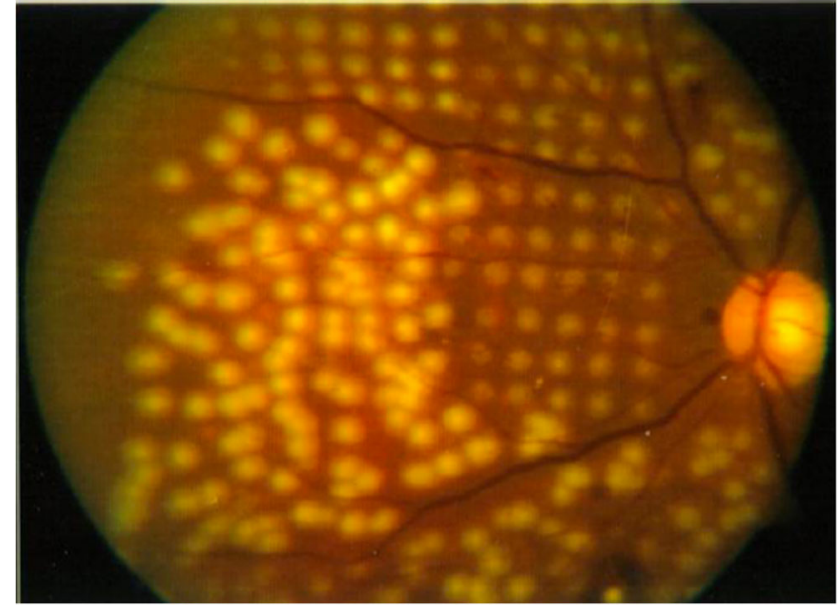

Fig. 1 Fundus photograph comparing conventional laser (lower left) and patterned scanning laser (upper right), demonstrating more uniformly spaced, small, and less intense spots provided by the pattern scanning laser. Reprinted with permission from Paulus, Y. M., Palanker, D., \& Blumenkranz, M. S. (2010). Short-pulse laser treatment: redefining retinal therapy. Retinal Physician, 7(1), 54-56

pulse durations result in less patient discomfort due to reduced heat diffusion into the choroid [47]. Optimization of laser wavelength along with spatial and temporal modulation of the laser beam can also be considered to maximize clinical utility while minimizing damage to surrounding tissue $[48$, 49].

Pattern scanning laser is commonly used for PRP in the treatment of PDR with similar clinical efficacy compared to conventional laser therapy. Pattern scanning laser parameters for PRP include spot size of 200um with duration of 10 to $20 \mathrm{~ms}$ placed just outside the arcades ( 1 disc diameter or more from the arcades), at least three disc diameters temporal to the macula, and at least one disc diameter nasal to the optic disc with patterns varying from $3 \times 3$ to $7 \times 7$ laser spot arrays. The outcomes of PRP performed with the pattern scanning system have been compared to PRP with conventional laser in several studies. For example, a 532-nm solid-state green laser (GLX) was compared to a multi-spot 532-nm pattern scanning laser approach in PRP treatment in a prospective, randomized clinical trial to compare the efficacy, collateral damage, and convenience of these PRP approaches [50]. This study demonstrated that pattern scanning laser resulted in less collateral tissue damage and similar regression of retinopathy compared to the GLX laser, and it was less time consuming and less painful for patients. However, in a separate study, the pattern scanning laser was reported to be less effective compared to conventional treatment in the treatment of high-risk PDR when applying equivalent number of laser treatment spots [51], although subsequently it was shown that the patients undergoing pattern scanning treatment in this study received significantly less treatment than the conventional laser group [52]. 
Pattern scanning laser is also a highly utilized method for the treatment of diabetic macular edema. Convenient laser pattern templates may be used for macular photocoagulation that include ring and arc patterns with a central foveal exclusion zone, ensuring that no laser burn is placed closer than a preset distance from the center of the foveal avascular zone. Pattern scanning is also utilized for "subthreshold" focal-grid laser in macular edema, with the goal of avoiding and preventing the enlargement of laser photocoagulation scars over time after treatment [53]. These approaches are highly effective for the treatment of macular edema, and in 2012 a large, retrospective observational case series reported that clinical and visual outcomes of short-pulse duration laser settings with the pattern scanning system were comparable to those of conventional argon laser parameters for the treatment of diabetic macular edema [54].

\section{Selective Retinal Therapy}

As noted above, conventional retinal photocoagulation is limited in its use for macular conditions because of the risk of vision loss from central scars (resulting in scotomas) and expansion of the laser scar over time. Selective retinal therapy (SRT) with microsecond pulses that have a shorter duration than the time needed for produced heat to diffuse was developed as an alternative laser modality, specifically with the goal of treating macular diseases that result from RPE dysfunction, including age related macular degeneration, DME, and central serous chorioretinopathy. Given the very short pulse duration used in SRT, the high temperature is confined primarily to the melanosomes inside RPE cells, which absorb approximately $50 \%$ of the incident green light [55]. This enables the selective treatment of the RPE cells without damage to the overlying photoreceptors, neurosensory retina, and choroid. There are two SRT modalities: a pulsed and continuous wave scanning mode, and a variety of clinical trials have validated the safety and efficacy of SRT in DME, central serous chorioretinopathy, and macular edema secondary to branch retinal vein occlusions [56-58]. For example, a prospective, two-center interventional uncontrolled pilot study of SRT as a treatment of CSME demonstrated statistically significant improvement in the mean best-corrected visual acuity in treated patients at 6-month follow-up, with no adverse effects [59]. However, SRT has not yet been commercialized or implemented for routine clinical use despite promising initial results, in part because it is challenging for physicians to use clinically given the lack of visible changes in the retinal appearance when applying laser spots, making it difficult to define the energy required for selective and therapeutic RPE damage [60].

\section{Subthreshold Diode Micropulse Laser}

Subthreshold diode micropulse (SDM) laser is another novel laser modality for photocoagulation designed to minimize collateral tissue damage for treatment of the macula. Similar to SRT, the goal of SDM is to provide selective therapeutic targeting of the RPE while sparing of the neurosensory retina, utilizing a near-infrared diode laser $(810 \mathrm{~nm})$ with bursts of submillisecond pulses [61, 62]. As the name implies, the term "subthreshold" refers to laser energy applied with no visible intra-retinal damage or scarring, either during or after treatment. Although the exact mechanism by which SDM induces a therapeutic response is not understood, it is hypothesized that SDM may alter the metabolic activity of the RPE, resulting in the release of cytokines that regulate angiogenesis and vascular leakage without any associated retinal damage [63-65]. SDM is delivered as microsecond laser pulses with variable intervals without laser treatment in order to allow the tissue to return to baseline temperature between pulses [66, 67], and it may be utilized in a low-intensity/high-density approach for the complete and confluent treatment of an area of diseased retina, such as an area of central macular edema [68]. In fact, the most widely used application of SDM is for the treatment of clinically significant macular edema (CSME) [69], and it has been shown to have a long-term effect on visual acuity and resolution of macular edema in a 3-year follow-up case series of 25 treated eyes [70]. In addition, SDM has been compared directly to the ETDRS or the modified ETDRS focal laser protocols for the treatment of DME in several randomized clinical trials; SDM was found to be equal or superior to modified ETDRS laser photocoagulation with less associated RPE damage for the treatment of DME [71, 72].

\section{Upcoming Innovative Clinical Laser and Delivery Platforms}

In addition to the efficacious novel laser approaches outlined above (scanning pattern laser, selective retinal therapy, subthreshold diode micropulse, etc.), several other important clinical innovations are likely to become main-stream in busy retina practices over the next few years, including endpoint management and image-guided navigated laser delivery. Table 1 compares the parameters and indications for several of these novel laser techniques.

Endpoint management refers to a modified laser therapy approach designed to precisely control laser energy relative to titration level [73], and this titration algorithm is commercially available for the 532-nm and 577-nm Pattern scanning lasers to provide highly predictable laser dosimetry based on the clinical indication and setting. The Endpoint Management algorithm is based upon titrating laser power to that needed to 
Table 1 Comparison of novel retinal laser techniques with indications for the treatment of PDR and DME

\begin{tabular}{|c|c|c|c|c|}
\hline & $\begin{array}{l}\text { Pattern scanning } \\
\text { (i.e., PASCAL®) }\end{array}$ & $\begin{array}{l}\text { Navigated laser } \\
\text { (i.e., NAVILAS®) }\end{array}$ & SDM & SRT \\
\hline $\begin{array}{l}\text { Laser } \\
\text { devices }\end{array}$ & $\begin{array}{l}\text { 532-nm } \\
\text { Nd-YAG/514-nm } \\
\text { argon laser }\end{array}$ & 577-nm yellow laser & 810-nm diode laser & $\begin{array}{l}\text { 527-nm Nd-YLF/532-nm } \\
\text { Nd-YAG laser }\end{array}$ \\
\hline $\begin{array}{l}\text { Pulse } \\
\text { duration }\end{array}$ & $10-1000 \mathrm{~ms}$ & $10-1000 \mathrm{~ms}$ & $100-300 \mu \mathrm{s}$ & $1.7 \mu \mathrm{s} / 15-60 \mathrm{~ms}$ \\
\hline Indications & PDR/DME & PDR/DME & DME & DME \\
\hline Advantages & $\begin{array}{l}\text { Shorter treatment } \\
\text { times, increased } \\
\text { safety }\end{array}$ & $\begin{array}{l}\text { Eye tracking, improved accuracy } \\
\text { and safety }\end{array}$ & Minimize collateral tissue damage & $\begin{array}{l}\text { Selectively damage RPE } \\
\text { cells }\end{array}$ \\
\hline Limitations & $\begin{array}{l}\text { Uncontrolled eye } \\
\text { movements }\end{array}$ & $\begin{array}{l}\text { No stereoscopic view, cannot } \\
\text { integrate ICG angiography }\end{array}$ & $\begin{array}{l}\text { Longer treatment time, treatment protocols are } \\
\text { not well established or standardized }\end{array}$ & $\begin{array}{l}\text { Inability to detect or } \\
\text { visualize treatment } \\
\text { effects }\end{array}$ \\
\hline
\end{tabular}

generate a barely visible retinal burn (defined as $100 \%$ nominal energy level), and then additional pulse energies can be utilized as a percentage of the nominal energy level in order to provide a spectrum of clinical laser intensities, from subvisible retinal laser to intense coagulative tissue effects. Subthreshold PRP using an Endpoint Management algorithm has been directly compared to conventional pattern scanning PRP for the treatment of severe non-proliferative diabetic retinopathy in a prospective study with regards to the rate of progression to PDR in 12-month follow-up, and Endpoint Management was found to be noninferior to conventional threshold pattern scanning PRP [74].

Image-guided laser therapies, such as "Navigated laser (NAVILAS)," are commercially available systems that utilize fundus imaging and treatment device for specific, targeted retinal laser photocoagulation in a pre-determined and highly precise manner. It can incorporate various imaging modalities such as infrared images, color fundus photographs, and fluorescein angiography images and utilize these to create detailed treatment plans for focal or large treatment areas with high reproducibility and precision. NAVILAS has been shown to be safe and effective in the treatment of DME with associated improvement in visual acuity and macular edema 12 months after treatment [75, 76] in a highly time-efficient manner [77], and has also been used for PRP treatment [78]. Nanosecond pulse duration laser synchronized with concurrent focused ultrasound, termed photo-mediated ultrasound therapy [79, 80], has also been described and used to treat clinically relevant animal models of retinal neovascularization without damaging surrounding tissues [81].

\section{Retinal Laser for PDR and DME Compared and Combined with Pharmacologic Therapies}

Since the development and wide-spread use of anti-VEGF agents for the treatment of PDR and DME, a number of important clinical trials have studied the efficacy of pharmacologic therapy alone compared to retinal photocoagulation, or pharmacologic therapy combined with retinal laser therapy, for these conditions. Similarly, additional studies have evaluated the role of intravitreal steroid therapy in the management of DME relative to photocoagulation, or in combination with laser treatment. The DRCR Retina Network (DRCR.net) has coordinated many of these studies, which are summarized briefly in Table 2. Only a subset of the DRCR Retina Network protocols most relevant to the scope of this review are included in Table 2, but a complete list is available on the DRCR website, including a number of studies that evaluated only anti-VEGF agents without a laser comparison group (https://public.jaeb.org/drcrnet/stdy).

DRCR Protocol $\mathrm{H}$ was a Phase 2, randomized, multi-center clinical trial to evaluate the efficacy of anti-VEGF therapy (bevacizumab) for DME, either as primary treatment or in combination with macular photocoagulation in patients 18 years or older [83]. Study eyes were randomly assigned to one of five groups: (1) Laser photocoagulation at baseline (with option for intravitreal injection if DME was present at 12-week follow-up), (2) $1.25 \mathrm{mg}$ intravitreal injection of bevacizumab at baseline and 6 weeks, (3) $2.5 \mathrm{mg}$ intravitreal injection of bevacizumab at baseline and 6 weeks, (4) $1.25 \mathrm{mg}$ intravitreal injection of bevacizumab at baseline with sham injection at 6 weeks, or (5) $1.25 \mathrm{mg}$ intravitreal injection of bevacizumab at baseline, laser photocoagulation at 3 weeks, and intravitreal injection of $1.25 \mathrm{mg}$ bevacizumab at 6 weeks. The main study conclusion after 70 weeks of follow-up was that intravitreal bevacizumab can effectively reduce DME in some eyes, and there was no apparent short-term benefit or adverse outcome when intravitreal bevacizumab was combined with focal photocoagulation [83]. DRCR Protocol I was a randomized clinical trial evaluating the effect of prompt versus deferred (for $\geq 24$ weeks) foal/grid laser treatment in eyes treated with intravitreal $0.5 \mathrm{mg}$ ranibizumab for DME [84]. A 3-year follow-up study of the Protocol I subjects 


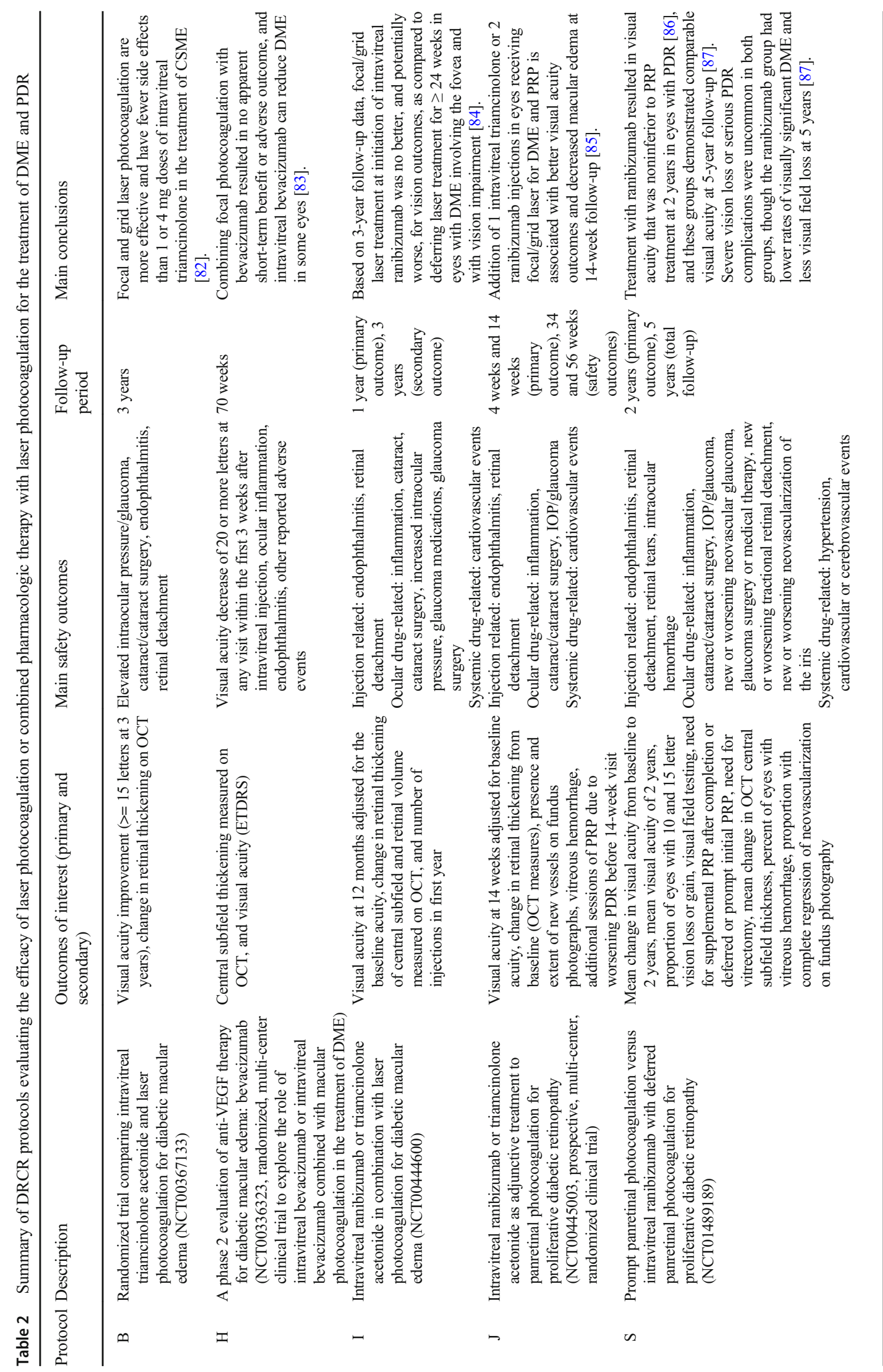


suggested that focal/grid laser treatment at initiation of intravitreal ranibizumab was no better, and potentially worse, for vision outcomes, as compared to deferring laser treatment for $\geq 24$ weeks in eyes with DME involving the fovea and with vision impairment [84]. DRCR Protocol J was a randomized clinical trial evaluating the short-term effects (14 weeks) of intravitreal ranibizumab or triamcinolone acetonide on macular edema following focal/grid laser for DME in eyes also receiving PRP [85]. This study found that the addition of 1 intravitreal triamcinolone or 2 ranibizumab injections in eyes receiving focal/grid laser for DME and PRP was associated with better visual acuity outcomes and decreased macular edema at 14-week follow-up [85]. Finally, DRCR Protocol $\mathrm{S}$ was a Phase III, prospective, multi-center randomized clinical trial evaluating the effect of prompt PRP versus intravitreal ranibizumab with deferred PRP for eyes with PDR. Treatment with ranibizumab resulted in visual acuity that was noninferior to PRP treatment at 2 years in eyes with PDR [86], and these groups demonstrated comparable visual acuity at 5-year follow-up [87]. Severe vision loss or serious PDR complications were uncommon in both groups, though the ranibizumab group had lower rates of visually significant DME and less visual field loss at 5 years [87].

In addition to the DRCR protocols, a number of other studies have investigated the efficacy of combined pharmacologic and laser therapy for PDR or DME. PRP laser alone versus a combination of intravitreal aflibercept and PRP treatment was evaluated in a retrospective study of 72 eyes with high-risk PDR [88]. There were no significant differences in bestcorrected visual acuity, central foveal thickness, and microaneurysms in the laser group before and after treatment, but there were statistically significant improvements in the combination therapy group compared to baseline. The differences between best-corrected visual acuity, central foveal thickness, and microaneurysms were statistically significantly different between the PRP only group and the combination therapy group, suggesting that combination therapy may provide improved morphologic and functional outcomes [88]. The efficacy and safety of anti-VEGF monotherapy (bevacizumab) versus combined anti-VEGF and subthreshold micropulse laser therapy for DME was evaluated in a retrospective study of 80 eyes, with the primary outcomes of interest defined as the mean number of required intravitreal injections, change of best-corrected visual acuity, and change in central macular thickness [89]. A significant increase in bestcorrected visual acuity was observed in the combined therapy group at 3, 6, 9, and 12 months of follow-up, whereas in the intravitreal monotherapy group, visual acuity was only significantly improved at month 3 . When compared to baseline, the decrease in central macular thickness was statistically significant in both groups at 3,6, 9, and 12-month follow-up. This study demonstrated that the use of combined intravitreal antiVEGF and subthreshold micropulse laser may be effective and safe for DME [89]. This conclusion was supported by a recent literature review of all studies utilizing a combination of subthreshold diode micropulse laser and intravitreal antiVEGF or steroid treatment for the management of DME, which reported that combination therapy resulted in fewer intravitreal injections that pharmacologic monotherapy with noninferior functional and morphologic outcomes [90].

Additional studies have evaluated the role of intravitreal steroid therapy in the management of DME relative to photocoagulation, including the DRCR Protocol B, which demonstrated that focal and grid laser photocoagulation are more effective and have fewer side effects than 1 or $4 \mathrm{mg}$ doses of intravitreal triamcinolone in the treatment of CSME [82]. Importantly, there are significant clinical considerations and possible undesired effects when laser photocoagulation and intravitreal steroid therapy are utilized concurrently, particularly relating to laser scar healing and residual tissue effects $[91,92]$. A rabbit model was utilized to evaluate the effect of intravitreal triamcinolone acetonide (TA) on the healing of retinal photocoagulation lesions using drug and laser dosing parameters typically used in the clinical setting [91], relative to control treatment with balanced salt solution injection rather than TA. While the TA treatment groups demonstrated significant reduction in retinal thickness and laser-induced edema compared to the balanced salt solution control eyes, this study demonstrated that TA injection previously or concurrently with photocoagulation interfered with retinal laser lesion healing, resulting in wider residual scarring that was especially notable in more intense laser burns.

\section{Considerations for pharmacologic therapy and Laser Photocoagulation "in Real World" Conditions}

Despite the well-established efficacy and benefits of intravitreal anti-VEGF therapy (alone or in combination with laser) for the treatment of PDR and DME, any treatment plan that relies on regular clinic visits and regular intravitreal injections is subject to failure if a patient cannot reliably return for care. A number of personal, social, financial, and medical constraints may limit a patient's ability to return for injections, including loss of insurance coverage, other critical illnesses, psycho/social factors, or (as recently demonstrated), concerns about seeking medical care during the COVID-19 pandemic [93-95]. Given these real-world constraints, laser treatment has the benefit of reducing the number of required injections while offering a highly effective, long-lasting therapeutic benefit $[96,97]$. Unfortunately, every retina provider has seen first-hand the possible devastating outcomes of untreated or incompletely treated PDR when a patient is lost to follow up, including permanent vision loss and complex surgical needs 
from neovascular glaucoma, tractional retinal detachments, and profound retinal nonperfusion and ischemia [98, 99].

\section{Conclusion}

Despite the widespread use and high efficacy of anti-VEGF therapy for diabetic retinopathy and diabetic macular edema, retinal laser photocoagulation remains a vital therapeutic method for the treatment of these conditions.

PRP laser treatment for PDR offers a definitive, durable treatment method to prevent severe vision-threatening complications such as neovascular glaucoma and tractional retinal detachments; it remains the gold standard for treatment of PDR. Importantly, patients who have complete PRP treatment are not reliant on serial intravitreal anti-VEGF injections for the treatment of the PDR, minimizing the risk of rare but devastating complications from post-injection endophthalmitis or worsening of their clinical disease in cases of loss-tofollow-up or inability to present for routine clinical care (such as during the peak of the COVID-19 pandemic, or loss of insurance coverage) [93]. Although PDR may theoretically be managed with regular anti-VEGF injections, PRP laser offers a life-long management plan in real-world settings in which patients cannot return to the clinic every 4-6 weeks. Similarly, focal and grid laser photocoagulation for the treatment of CSME can be highly effective and provide longstanding resolution of macular edema without the need for serial intravitreal injections, although the final best-corrected visual acuity in eyes treated with laser photocoagulation alone as compared to combination therapy may be lower in some cases.

In addition, a number of recent laser technology innovations and modifications to conventional laser photocoagulation have led to improved clinical efficacy with simultaneous reduction of side effects and adverse permanent vision consequences such as scotoma formation, choroidal neovascularization, retinal scarring, and patient discomfort. For example, pattern scanning laser has decreased the time required and the discomfort experienced by patients for PRP treatment of PDR. Selective retinal therapy (SRT) enables the localized, selective treatment of RPE cells while limiting collateral damage to the neurosensory retina. Subthreshold micropulse laser results in therapeutic effect without inducing any detectable intra-retinal tissue damage. These advances, along with other innovations such as Endpoint Management and Navigated Laser, have improved the safety, efficacy, and efficiency of retinal photocoagulation.

Given that intravitreal anti-VEGF and corticosteroid agents are now common therapeutic agents for the treatment of both PDR and DME, research is ongoing as to the best approaches and treatment paradigms for the combined use of laser and pharmacologic therapy. However, repeated and long-term intravitreal injections place a significant burden on the healthcare system, patients, and providers, and they are not without significant possible risks including endophthalmitis [100]. Continuing innovations in laser technology and improved understanding of laser-retinal interactions and pathophysiology make us think that laser therapy will continue to play a critical role in the treatment of diabetic retinopathy and diabetic macular edema for many years to come.

Author Contribution Y.M.P. is the corresponding author for the manuscript. All authors contributed to the design and drafting the paper and reviewed and approved the manuscript for scholarly content.

Funding L.E. is supported by the Heed Ophthalmic Foundation and the VitreoRetinal Surgery Foundation.

Y.M.P. is supported by the National Eye Institute (1K08EY027458, 1R41EY031219, 1R01EY029489), unrestricted departmental support from Research to Prevent Blindness, and Alliance for Vision Research, and Fight for Sight - International Retinal Research Foundation.

\section{Declarations}

Conflict of Interest Lesley Everett declares no conflict of interest. Yannis Paulus has patents through the University of Michigan, equity and patents licenses to PhotoSonoX LLC, and serves as a consultant on a Department of Defense grant with Hedgefog Research Inc evaluating retinal photocoagulation injuries of the retina.

Human and Animal Rights and Informed Consent This article does not contain any studies with human or animal subjects performed by any of the authors.

\section{References}

1. CR M. Lasers in opthalmology: past, present and future. J Mod Opt. 2003;50(15-17):2351-60.

2. CDC. Diabetic Retinopathy 2020 [3/21/21]. Available from: https://www.cdc.gov/visionhealth/pdf/factsheet.pdf.

3. Palmberg PF. Diabetic retinopathy. Diabetes. 1977;26(7):703-9.

4. Blumenkranz MS. The evolution of laser therapy in ophthalmology: a perspective on the interactions between photons, patients, physicians, and physicists: the LXX Edward Jackson Memorial Lecture. Am J Ophthalmol. 2014;158(1):12-25 e1.

5. Li J, Paulus YM. Advances in retinal laser therapy. Int $\mathrm{J}$ Ophthalmic Res. 2018;4(1):259-64.

6. Xie X, Liu Q, Paulus YM. Innovations in retinal laser technology. Optics and Photonics Journal. 2018;8(6):173-86. https://www. scirp.org/journal/OPJ/

7. Xie X, Liu Q, Paulus YM. Novel retinal laser therapies. Int J Ophthalmic Res. 2018;4(1):272-9.

8. WB B. Laser oscillation in singly ionized argon in the visible spectrum. Appl Phys Lett. 1964;4(7):128-30.

9. Birngruber R, Hillenkamp F, Gabel VP. Theoretical investigations of laser thermal retinal injury. Health Phys. 1985;48(6):781-96.

10. Lock JH, Fong KC. Retinal laser photocoagulation. Med J Malaysia. 2010;65(1):88-94 quiz 5.

11. Paulus YM, Jain A, Gariano RF, Stanzel BV, Marmor M, Blumenkranz MS, et al. Healing of retinal photocoagulation lesions. Invest Ophthalmol Vis Sci. 2008;49(12):5540-5. 
12. Wolbarsht ML, Landers MB 3rd. The rationale of photocoagulation therapy for proliferative diabetic retinopathy: a review and a model. Ophthalmic Surg. 1980;11(4):235-45.

13. Stefansson E. The therapeutic effects of retinal laser treatment and vitrectomy. A theory based on oxygen and vascular physiology. Acta Ophthalmol Scand. 2001;79(5):435-40.

14. Ascaso FJ, Huerva V, Grzybowski A. The role of inflammation in the pathogenesis of macular edema secondary to retinal vascular diseases. Mediat Inflamm. 2014;2014:432685.

15. Ogata N, Ando A, Uyama M, Matsumura M. Expression of cytokines and transcription factors in photocoagulated human retinal pigment epithelial cells. Graefes Arch Clin Exp Ophthalmol. 2001;239(2):87-95.

16. Arnarsson A, Stefansson E. Laser treatment and the mechanism of edema reduction in branch retinal vein occlusion. Invest Ophthalmol Vis Sci. 2000;41(3):877-9.

17. Wilson DJ, Finkelstein D, Quigley HA, Green WR. Macular grid photocoagulation. An experimental study on the primate retina. Arch Ophthalmol. 1988;106(1):100-5.

18. Lee CM, Olk RJ. Modified grid laser photocoagulation for diffuse diabetic macular edema. Long-term visual results. Ophthalmology. 1991;98(10):1594-602.

19. Olk RJ. Modified grid argon (blue-green) laser photocoagulation for diffuse diabetic macular edema. Ophthalmology. 1986;93(7): 938-50.

20. Olk RJ. Argon green (514 nm) versus krypton red (647 nm) modified grid laser photocoagulation for diffuse diabetic macular edema. Ophthalmology. 1990;97(9):1101-12 discussion 12-3.

21. Striph GG, Hart WM Jr, Olk RJ. Modified grid laser photocoagulation for diabetic macular edema. The effect on the central visual field. Ophthalmology. 1988;95(12):1673-9.

22. Little HL, Zweng HC, Peabody RR. Argon laser slit-lamp retinal photocoagulation. Trans Am Acad Ophthalmol Otolaryngol. 1970;74(1):85-97.

23. Law NM, Fan RF. Clinical experience with the laser indirect ophthalmoscope. Ann Acad Med Singap. 1991;20(6):750-4.

24. Smiddy WE. Diode endolaser photocoagulation. Arch Ophthalmol. 1992;110(8):1172-4.

25. Treatment techniques and clinical guidelines for photocoagulation of diabetic macular edema. Early Treatment Diabetic Retinopathy Study Report Number 2. Early Treatment Diabetic Retinopathy Study Research Group. Ophthalmology. 1987;94(7):761-74.

26. Prskavec FH, Fulmek R, Klemen C, Stelzer N. Changes in the visual field and dark adaptation following panretinal photocoagulation in diabetic retinopathy. Klin Monatsbl Augenheilkd. 1986;189(5):385-7.

27. Russell PW, Sekuler R, Fetkenhour C. Visual function after panretinal photocoagulation: a survey. Diabetes Care. 1985;8(1):5763.

28. Blondeau P, Pavan PR, Phelps CD. Acute pressure elevation following panretinal photocoagulation. Arch Ophthalmol. 1981;99(7):1239-41.

29. Irvine WD, Smiddy WE, Nicholson DH. Corneal and iris burns with the laser indirect ophthalmoscope. Am J Ophthalmol. 1990;110(3):311-3.

30. Lobes LA Jr, Bourgon P. Pupillary abnormalities induced by argon laser photocoagulation. Ophthalmology. 1985;92(2):234-6.

31. Photocoagulation treatment of proliferative diabetic retinopathy. Clinical application of Diabetic Retinopathy Study (DRS) findings, DRS Report Number 8. The Diabetic Retinopathy Study Research Group. Ophthalmology. 1981;88(7):583-600.

32. Four risk factors for severe visual loss in diabetic retinopathy. The third report from the Diabetic Retinopathy Study. The Diabetic Retinopathy Study Research Group. Arch Ophthalmol. 1979;97(4):654-5.
33. Photocoagulation for diabetic macular edema. Early Treatment Diabetic Retinopathy Study report number 1. Early Treatment Diabetic Retinopathy Study research group. Arch Ophthalmol. 1985;103(12):1796-806.

34. Patz ASR. The ETDRS and Diabetes 2000. Ophthalmology. 1991;98(5):739-840.

35. Early photocoagulation for diabetic retinopathy. ETDRS report number 9. Early Treatment Diabetic Retinopathy Study Research Group. Ophthalmology. 1991;98(5 Suppl):766-85.

36. Techniques for scatter and local photocoagulation treatment of diabetic retinopathy: Early Treatment Diabetic Retinopathy Study Report no. 3. The Early Treatment Diabetic Retinopathy Study Research Group. Int Ophthalmol Clin. 1987;27(4):254-64.

37. Photocoagulation for diabetic macular edema: Early Treatment Diabetic Retinopathy Study Report no. 4. The Early Treatment Diabetic Retinopathy Study Research Group. Int Ophthalmol Clin. 1987;27(4):265-72.

38. Roider J. Laser treatment of retinal diseases by subthreshold laser effects. Semin Ophthalmol. 1999;14(1):19-26.

39. Schatz H, Madeira D, McDonald HR, Johnson RN. Progressive enlargement of laser scars following grid laser photocoagulation for diffuse diabetic macular edema. Arch Ophthalmol. 1991;109(11):1549-51.

40. Friberg TR. Subthreshold (invisible) modified grid diode laser photocoagulation and diffuse diabetic macular edema (DDME). Ophthalmic Surg Lasers. 1999;30(9):705.

41. Writing Committee for the Diabetic Retinopathy Clinical Research N, Fong DS, Strauber SF, Aiello LP, Beck RW, Callanan DG, et al. Comparison of the modified Early Treatment Diabetic Retinopathy Study and mild macular grid laser photocoagulation strategies for diabetic macular edema. Arch Ophthalmol. 2007;125(4):469-80.

42. Romero-Aroca P, Reyes-Torres J, Baget-Bernaldiz M, BlascoSune C. Laser treatment for diabetic macular edema in the 21st century. Curr Diabetes Rev. 2014;10(2):100-12.

43. Blumenkranz MS, Yellachich D, Andersen DE, Wiltberger MW, Mordaunt D, Marcellino GR, et al. Semiautomated patterned scanning laser for retinal photocoagulation. Retina. 2006;26(3):370-6.

44. Paulus YM, Kaur K, Egbert PR, Blumenkranz MS, Moshfeghi DM. Human histopathology of PASCAL laser burns. Eye (Lond). 2013;27(8):995-6.

45. Jain A, Blumenkranz MS, Paulus Y, Wiltberger MW, Andersen DE, Huie $P$, et al. Effect of pulse duration on size and character of the lesion in retinal photocoagulation. Arch Ophthalmol. 2008;126(1):78-85.

46. Sher A, Jones BW, Huie P, Paulus YM, Lavinsky D, Leung LS, et al. Restoration of retinal structure and function after selective photocoagulation. J Neurosci. 2013;33(16):6800-8.

47. Muqit MM, Marcellino GR, Gray JC, McLauchlan R, Henson DB, Young LB, et al. Pain responses of Pascal $20 \mathrm{~ms}$ multi-spot and $100 \mathrm{~ms}$ single-spot panretinal photocoagulation: Manchester Pascal Study, MAPASS report 2. Br J Ophthalmol. 2010;94(11): 1493-8.

48. Sramek C, Leung LS, Leng T, Brown J, Paulus YM, Schuele G, et al. Improving the therapeutic window of retinal photocoagulation by spatial and temporal modulation of the laser beam. $\mathrm{J}$ Biomed Opt. 2011;16(2):028004.

49. Sramek CK, Leung LS, Paulus YM, Palanker DV. Therapeutic window of retinal photocoagulation with green $(532-\mathrm{nm})$ and yellow (577-nm) lasers. Ophthalmic Surg Lasers Imaging. 2012;43(4):341-7.

50. Nagpal M, Marlecha S, Nagpal K. Comparison of laser photocoagulation for diabetic retinopathy using 532-nm standard laser versus multispot pattern scan laser. Retina. 2010;30(3):452-8.

51. Chappelow AV, Tan K, Waheed NK, Kaiser PK. Panretinal photocoagulation for proliferative diabetic retinopathy: pattern scan 
laser versus argon laser. Am J Ophthalmol. 2012;153(1):137-42 e2.

52. Palanker D, Blumenkranz MS. Panretinal photocoagulation for proliferative diabetic retinopathy. Am J Ophthalmol. 2012;153(4):780-1 author reply 1-2.

53. Muqit MM, Gray JC, Marcellino GR, Henson DB, Young LB, Patton N, et al. Barely visible 10-millisecond pascal laser photocoagulation for diabetic macular edema: observations of clinical effect and burn localization. Am J Ophthalmol. 2010;149(6):979$86 \mathrm{e} 2$.

54. Muqit MM, Sanghvi C, McLauchlan R, Delgado C, Young LB, Charles SJ, et al. Study of clinical applications and safety for Pascal(R) laser photocoagulation in retinal vascular disorders. Acta Ophthalmol. 2012;90(2):155-61.

55. Gabel V-P BR, Hillenkamp F. Visible and near infrared light absorption in pigment epithelium and choroid. XXIII Concilium Ophthalmologicum. 1978;450:658-62.

56. Roider J, Brinkmann R, Wirbelauer C, Laqua H, Birngruber R. Subthreshold (retinal pigment epithelium) photocoagulation in macular diseases: a pilot study. Br J Ophthalmol. 2000;84(1): 40-7.

57. Paulus YM, Jain A, Nomoto H, Sramek C, Gariano RF, Andersen $\mathrm{D}$, et al. Selective retinal therapy with microsecond exposures using a continuous line scanning laser. Retina. 2011;31(2):380-8.

58. Brinkmann R, Roider J, Birngruber R. Selective retina therapy (SRT): a review on methods, techniques, preclinical and first clinical results. Bull Soc Belge Ophtalmol. 2006;302:51-69.

59. Roider J, Liew SH, Klatt C, Elsner H, Poerksen E, Hillenkamp J, et al. Selective retina therapy (SRT) for clinically significant diabetic macular edema. Graefes Arch Clin Exp Ophthalmol. 2010;248(9): 1263-72.

60. Brinkmann R, Schüle G, Joachimmeyer E, Roider J, Birngruber $\mathrm{R}$, "Determination of absolute fundus temperatures during retinal laser photocoagulation and selective RPE treatment," Invest. Ophthalmol. Visual Sci. (2001);42:696.

61. Paulus YM. Short-pulse laser treatment: redefining retinal therapy. Retin Physician. 2010;2011:1-9.

62. Pankratov M P. Pulsed delivery of laser energy in experimental thermal retinal photocoagulation. Proceedings Volume 1202, Laser-Tissue Interaction. 1990. https://doi.org/10.1117/12.17626

63. Flaxel C, Bradle J, Acott T, Samples JR. Retinal pigment epithelium produces matrix metalloproteinases after laser treatment. Retina. 2007;27(5):629-34.

64. Hattenbach LO, Beck KF, Pfeilschifter J, Koch F, Ohrloff C, Schacke W. Pigment-epithelium-derived factor is upregulated in photocoagulated human retinal pigment epithelial cells. Ophthalmic Res. 2005;37(6):341-6.

65. Mainster MA. Decreasing retinal photocoagulation damage: principles and techniques. Semin Ophthalmol. 1999;14(4):200-9.

66. Sivaprasad S, Elagouz M, McHugh D, Shona O, Dorin G. Micropulsed diode laser therapy: evolution and clinical applications. Surv Ophthalmol. 2010;55(6):516-30.

67. Kiire CSS, Chong V. Subthreshold micropulse laser therapy for retinal disorders. Retina Today. 2011;JANUARY-FEBRUARY: 67-70. https://retinatoday.com/articles/2011-jan/subthresholdmicropulse-laser-therapy-forretinal-disorders

68. Su D, Hubschman JP. A review of subthreshold micropulse laser and recent advances in retinal laser technology. Ophthalmol Therapy. 2017;6(1):1-6.

69. Luttrull JK, Dorin G. Subthreshold diode micropulse laser photocoagulation (SDM) as invisible retinal phototherapy for diabetic macular edema: a review. Curr Diabetes Rev. 2012;8(4):274-84.

70. Sivaprasad S, Sandhu R, Tandon A, Sayed-Ahmed K, McHugh DA. Subthreshold micropulse diode laser photocoagulation for clinically significant diabetic macular oedema: a three-year follow up. Clin Exp Ophthalmol. 2007;35(7):640-4.
71. Figueira J, Khan J, Nunes S, Sivaprasad S, Rosa A, de Abreu JF, et al. Prospective randomised controlled trial comparing subthreshold micropulse diode laser photocoagulation and conventional green laser for clinically significant diabetic macular oedema. Br J Ophthalmol. 2009;93(10):1341-4.

72. Lavinsky D, Cardillo JA, Melo LA Jr, Dare A, Farah ME, Belfort R Jr. Randomized clinical trial evaluating mETDRS versus normal or high-density micropulse photocoagulation for diabetic macular edema. Invest Ophthalmol Vis Sci. 2011;52(7):4314-23.

73. Lavinsky D, Sramek C, Wang J, Huie P, Dalal R, Mandel Y, et al. Subvisible retinal laser therapy: titration algorithm and tissue response. Retina. 2014;34(1):87-97.

74. Lai, K., et al. Subthreshold Pan-Retinal Photocoagulation Using Endpoint Management Algorithm for Severe Nonproliferative Diabetic Retinopathy: A Paired Controlled Pilot Prospective Study. Ophthalmic Res. 2021;64(4):648-655.

75. Jung JJ, Gallego-Pinazo R, Lleo-Perez A, Huz JI, Barbazetto IA. NAVILAS laser system focal laser treatment for diabetic macular edema-one year results of a case series. Open Ophthalmol J. 2013;7:48-53.

76. Kozak I, Oster SF, Cortes MA, Dowell D, Hartmann K, Kim JS, et al. Clinical evaluation and treatment accuracy in diabetic macular edema using navigated laser photocoagulator NAVILAS. Ophthalmology. 2011;118(6):1119-24.

77. Ober MD, Kernt M, Cortes MA, Kozak I. Time required for navigated macular laser photocoagulation treatment with the Navilas. Graefes Arch Clin Exp Ophthalmol. 2013;251(4):1049-53.

78. Kernt M, Cheuteu R, Vounotrypidis E, Haritoglou C, Kampik A, Ulbig MW, et al. Focal and panretinal photocoagulation with a navigated laser (NAVILAS(R)). Acta Ophthalmol. 2011;89(8): e662-4.

79. Hu Z, Zhang H, Mordovanakis A, Paulus YM, Liu Q, Wang X, et al. High-precision, non-invasive anti-microvascular approach via concurrent ultrasound and laser irradiation. Sci Rep. 2017;7: 40243.

80. Zhang H, Xie X, Li J, Qin Y, Zhang W, Cheng Q, et al. Removal of choroidal vasculature using concurrently applied ultrasound bursts and nanosecond laser pulses. Sci Rep. 2018;8(1):12848.

81. Paulus YM, Qin Y, Yu Y, Fu J, Wang X, Yang X. Photo-mediated ultrasound therapy to treat retinal neovascularization. Annu Int Conf IEEE Eng Med Biol Soc. 2020;2020:5244-7.

82. DRCR N. A randomized trial comparing intravitreal triamcinolone acetonide and focal/grid photocoagulation for diabetic macular edema. Ophthalmology Ophthalmology. 2008;115(9):1447145010.

83. Diabetic Retinopathy Clinical Research N, Scott IU, Edwards AR, Beck RW, Bressler NM, Chan CK, et al. A phase II randomized clinical trial of intravitreal bevacizumab for diabetic macular edema. Ophthalmology. 2007;114(10):1860-7.

84. Diabetic Retinopathy Clinical Research N, Elman MJ, Qin H, Aiello LP, Beck RW, Bressler NM, et al. Intravitreal ranibizumab for diabetic macular edema with prompt versus deferred laser treatment: three-year randomized trial results. Ophthalmology. 2012;119(11):2312-8.

85. Diabetic Retinopathy Clinical Research N, Googe J, Brucker AJ, Bressler NM, Qin H, Aiello LP, et al. Randomized trial evaluating short-term effects of intravitreal ranibizumab or triamcinolone acetonide on macular edema after focal/grid laser for diabetic macular edema in eyes also receiving panretinal photocoagulation. Retina. 2011;31(6):1009-27.

86. Writing Committee for the Diabetic Retinopathy Clinical Research N, Gross JG, Glassman AR, Jampol LM, Inusah S, Aiello LP, et al. Panretinal photocoagulation vs intravitreous ranibizumab for proliferative diabetic retinopathy: a randomized clinical trial. JAMA. 2015;314(20):2137-46. 
87. Gross JG, Glassman AR, Liu D, Sun JK, Antoszyk AN, Baker $\mathrm{CW}$, et al. Five-year outcomes of panretinal photocoagulation vs intravitreous ranibizumab for proliferative diabetic retinopathy: a randomized clinical trial. JAMA Ophthalmol. 2018;136(10): $1138-48$.

88. Tao, Y., et al. Retrospective study of aflibercept in combination therapy for high-risk proliferative diabetic retinopathy and diabetic maculopathy. Int Ophthalmol. 2021 Jun;41(6):2157-2165.

89. Altinel, MG., et al. Comparison of the efficacy and safety of antiVEGF monotherapy versus anti-VEGF therapy combined with subthreshold micropulse laser therapy for diabetic macular edema. Lasers Med Sci. 2021 Sep;36(7):1545-1553.

90. Gawecki M. Subthreshold diode micropulse laser combined with intravitreal therapy for macular edema-a systematized review and critical approach. J Clin Med. 2021;10(7).

91. Nomoto H, Lavinsky D, Paulus YM, Leung LS, Dalal R, Blumenkranz MS, et al. Effect of intravitreal triamcinolone acetonide on healing of retinal photocoagulation lesions. Retina. 2013;33(1):63-70.

92. Dot C, Behar-Cohen F, BenEzra D, Doat M, Jonet L, May F, et al. Influence of triamcinolone intravitreal injection on retinochoroidal healing processes. Exp Eye Res. 2007;84(6):1081-9.

93. Lindeke-Myers, A., et al. Patient Perceptions of SARS-CoV-2 Exposure Risk and Association with Continuity of Ophthalmic Care. JAMA Ophthalmol. 2021 May 1;139(5):508-515.
94. Corazza P, D'Alterio FM, Younis S. Proposed algorithm during COVID-19 pandemic for patient management in medical retina clinic. Int J Retina Vitreous. 2020;6:20.

95. Yang KB, Feng H, Zhang H. Effects of the COVID-19 pandemic on anti-vascular endothelial growth factor treatment in China. Front Med (Lausanne). 2020;(7):576275.

96. DA Paulus YM. A conversation with Dr. Paulus, MD, FACS, on Endpoint Management ${ }^{\mathrm{TM}}$ during COVID. Retina Today. 2020;15(7). https://retinatoday.com/pdfs/1020_us.pdf

97. Moussa, M., et al. Intravitreal injections of anti-VEGF agents during COVID-19 pandemic: clinical audit from Tanta University Hospital. Int Ophthalmol. 2021 Apr;41(4):1437-1443.

98. Obeid A, Su D, Patel SN, Uhr JH, Borkar D, Gao X, et al. Outcomes of eyes lost to follow-up with proliferative diabetic retinopathy that received panretinal photocoagulation versus intravitreal anti-vascular endothelial growth factor. Ophthalmology. 2019;126(3):407-13.

99. Wubben TJ, Johnson MW, Anti VTISG. Anti-vascular endothelial growth factor therapy for diabetic retinopathy: consequences of inadvertent treatment interruptions. Am J Ophthalmol. 2019;204: 13-8.

100. Kim LA, D'Amore PA. A brief history of anti-VEGF for the treatment of ocular angiogenesis. Am J Pathol. 2012;181(2):376-9.

Publisher's Note Springer Nature remains neutral with regard to jurisdictional claims in published maps and institutional affiliations. 